\author{
I J M I \\ International Journal of Machine Intelligence \\ ISSN: 0975-2927 \& E-ISSN: 0975-9166, Volume 3, Issue 3, 2011, pp-103-107 \\ Available online at http://www.bioinfo.in/contents.php?id=31
}

\title{
IRIS CODE GENERATION AND RECOGNITION
}

\section{GITE H.R., MAHENDER C.N.}

Department of Computer Science and Information Technology, Dr. Babasaheb Ambedkar Marathwada University, Aurangabad (MS), India-431002.

*Corresponding Author: Email- hanumantgite@yahoo.com, nam.mah@gmail.com

\section{Received: September 29, 2011; Accepted: November 03, 2011}

Abstract- Iris is one of the important biometrics techniques. The pupil may vary in from $10 \%$ to $80 \%$ of the iris diameter, but fundamental rods \& cons structure for an individual remains unique till the life time. The important thing is its feature which has a unique pattern and not related to genetic feature. In our work we have localized, normalized and enhanced the image by using Daugman's Integro-differential Operator, Cartesian to polar coordinates, Histogram equalization, reflectively Gaussian filter was applied and then iris code were generated. Hamming distance was used for pattern matching. The overall success rate achieved $80 \%$.

Key words - Iris, Pattern, Hamming Distance, Iris Code.

\section{Introduction}

Biometrics is the science of automated recognition of persons based on one or more physiological or behavioral characteristics. Biometrics adds a unique identifier to network authentications, once done it's extremely difficult to duplicate. Smart cards \& tokens also provide a unique identifier, but biometrics has an advantage over these devices, that is a use can't lose or forget his or her fingerprint, retina or voice.

Human iris is taken as one of the Biometric element. The iris is a thin circular diaphragm, which lies between the cornea and the lens of the human eye. A front-on view of the iris is shown in Fig (1). The iris is perforated close to its centre by a circular aperture known as the pupil. The function of the iris is to control the amount of light entering through the pupil, and this is done by the sphincter and the dilator muscles, which adjust the size of the pupil. The average diameter of the iris is $12 \mathrm{~mm}$, and the pupil size can vary from $10 \%$ to $80 \%$ of the iris diameter [2, 3].

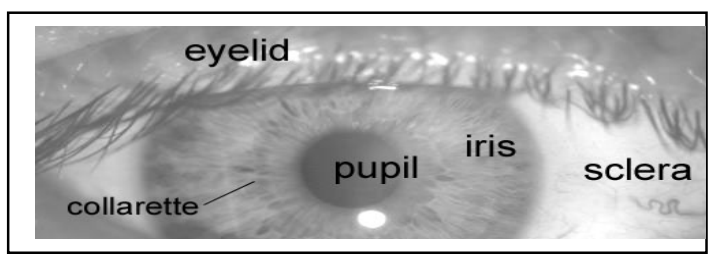

Fig. (1) A front-on view of the human eye.

The iris consists of a number of layers; the lowest is the epithelium layer, which contains dense pigmentation cells. The stromal layer lies above the epithelium layer, and contains blood vessels, pigment cells and the two iris muscles. The density of stromal pigmentation determines the color of the iris. The externally visible surface of the multi-layered iris contains two zones, which often differ in color [3].

Formation of the iris begins during the third month of embryonic life[3]. The unique pattern on the surface of the iris is formed during the first year of life, and pigmentation of the stroma takes place for the first few years [9]. Formation of the unique patterns of the iris is random and not related to any genetic factors [4]. The only characteristic that is dependent on genetics is the pigmentation of the iris, which determines its color. Due to the epigenetic nature of iris patterns, the two eyes of an individual contain completely independent iris patterns, and identical twins possess uncorrelated iris patterns.

\section{Iris Recognition System}

The iris is an externally visible, yet protected organ whose unique epigenetic pattern remains stable throughout adult life. These characteristics make it very attractive for use as a biometric for identifying individuals. The biometric template contains an objective mathematical representation of the unique information stored in the iris, and allows comparisons to be made between templates. When a subject wishes to be identified by iris recognition system, their eye is first photographed, and then a template created for their iris region. This template is then compared with the other templates stored in a database until either a matching template is found and the subject is identified, or no match is found and the subject remains unidentified.

Compared with other biometric technologies, such as face, speech and finger recognition, iris recognition can 
easily be considered as the most reliable form of biometric technology. However, there have been no independent trials of the technology [1], and source code for systems is not available. Also, there is a lack of publicly available datasets for testing and research, and the test results published have usually been produced using carefully imaged irises under favorable condition.

\section{Architecture of iris system}

The iris identification system is divided into four main functional blocks: First acquisition of human eye .Then, the images are pre-processed to normalize the scale and illumination of the iris and localize the iris pattern followed by feature extraction and finally decision is made by the means of matching.

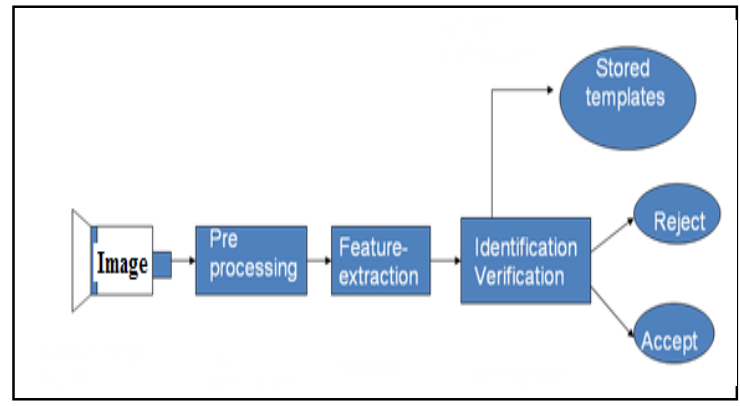

Fig. (2) Iris Recognition System

\section{Database Information}

In our work we have used MMU (Multimedia University) Database [17]. The latest threats of security have led to the increased awareness of biometric technologies. Iris recognition is one of the most secure biometric approaches as it is non-invasive and stable throughout life. Moreover, it does not require physical contact with the camera. In this way, the hygienic issue is minimized. Generally, there are four major processes for a particular iris recognition system. They are image acquisition, iris pre-processing, feature extraction and matching.

MMU iris database contributes a total number of 450 iris images which were taken using LG IrisAccess ${ }^{\circledR} 2200$. This camera is semi-automated and it operates at the range of $7-25 \mathrm{~cm}$. On the other hand, MMU1 iris database consists of 995 iris images. The iris images are collected using Panasonic BM-ET100US Authenticam and its operating range is even farther with a distance of $47-53 \mathrm{~cm}$ away from the user. These iris images are contributed by 100 volunteers with different age and nationality. They come from Asia, Middle East, Africa and Europe. Each of them contributes 5 iris images for each eye. There are 5 left eye iris images which are excluded from the database due to cataract disease [15] [17].

\section{Preprocessing}

The acquired image always contains not only the "useful" parts (IRIS) but also some parts (e.g. eyelid, pupil \& reflection) which are not useful for our work. Under some conditions, the brightness is not uniformly distributed. In addition, different eye-to-camera distance may result in different image sizes of the same eye. For the purpose of analysis, the original image needs to be processed. The preprocessing in our work composed of three steps:

\section{Iris Localization}

We have used Daugman's Integro-differential Operator for localization. Daugman's algorithm is based on applying an integro-differential operator to find the iris and pupil contour [3].

$$
\max \left(r, x_{0}, y_{0}\right) \mid G_{\sigma}(r) * \frac{\partial}{\partial r} \oint_{r, x_{0}, y_{0}} \frac{I(x, y)}{2 \prod r} d s
$$

Equation. Daugman's Integro-Differential Equation

Where: $x_{0}, y_{0}, r_{0}$ the center and radius of coarse circle (for each of pupil and iris). $G_{\sigma}(r)$ Gaussian function. $\Delta_{r}$ : The radius range for searching for. $\mathrm{I}(\mathrm{x}, \mathrm{y})$ the original iris image. $G_{\sigma}(r)$ Is a smoothing function, the smoothed image is then scanned for a circle that has a maximum gradient change, which indicates an edge. Eyelids are localized in a similar manner, with the path of contour integration changed from circular to an arc.

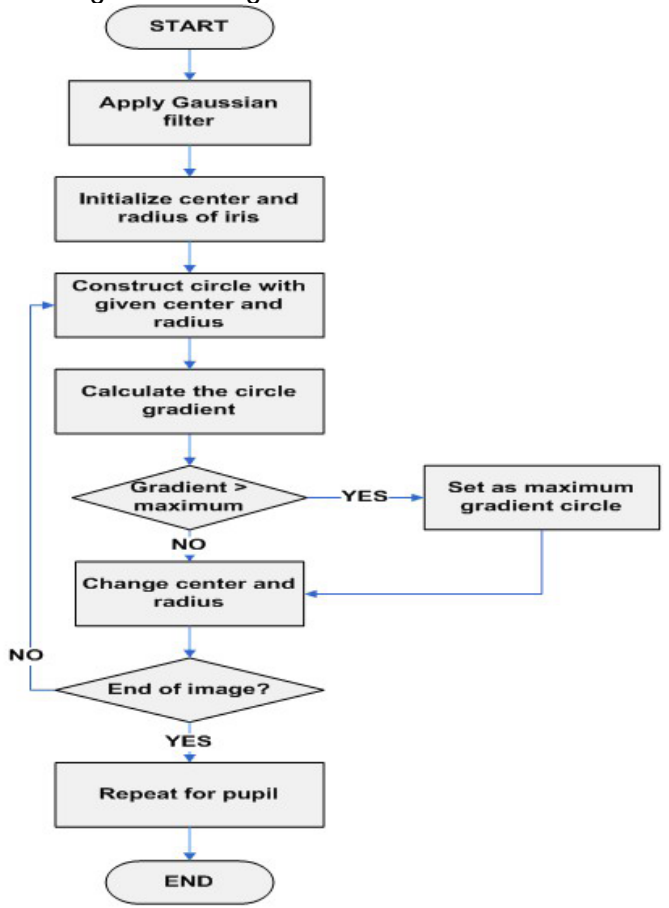

Fig. (3)Flow Chart for Daugman's Integro-differential operator

Problem was in determining the pupil boundary the maximum change should occur at the edge between the very dark pupil and the iris, which is relatively darker than the bright spots of the illumination

As a solution to this problem, modification to the integrodifferential operator is proposed to ignore all circles if any pixel on this circle has a value higher than a certain threshold. This threshold is determined to be 200 for the grayscale image. This ensures that only the bright spots - values usually higher than 245 - will be cancelled [8] [12]. 
Another solution we considered is to treat the illumination by truncating pixels higher than a certain threshold bright spots - to black. But this method failed in many images, this is because when the spot hits the pupil the illumination spreads on the pupil so as we treat the illumination spots it will leave behind a maximum changed edges that cannot be determined and the operator will consider it as the pupil boundary.

The integro-differential can be seen as a variation of the Hough transform, since it too makes use of first derivatives of the image and performs a search to find geometric parameters. Since it works with raw derivative information, it does not suffer from the thresholding problems of the Hough transform. However, the algorithm can fail where there is noise in the eye image, such as from reflections, as it works only on a local scale.

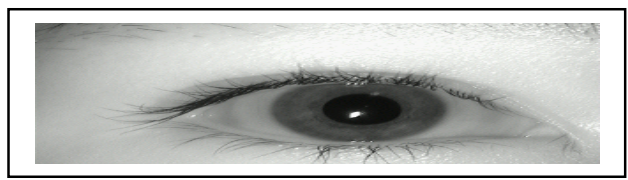

Fig .(4) Input Image

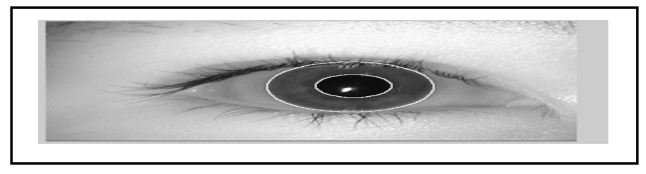

Fig. (5)Image after Localization by Integro-differential operator

\section{Normalization}

Daugman's normalization method transforms a localized iris texture from Cartesian to polar coordinates. The proposed method is capable of compensating the unwanted variations due to distance of eye from camera (scale) and its position with respect to the camera (translation). The Cartesian to polar transform is defined as:

$$
\begin{aligned}
& \text { Where } \\
& \begin{array}{l}
x_{p}(\theta)=x_{p 0}(\theta)+r_{p} * \cos (\theta), \\
y_{p}(\theta)=y_{p 0}(\theta)+r_{p} * \sin (\theta), \\
x_{i}(\theta)=x_{i 0}(\theta)+r_{i} * \cos (\theta), \\
y_{i}(\theta)=y_{i 0}(\theta)+r_{i} * \sin (\theta) .
\end{array}
\end{aligned}
$$

The process is inherently dimensionless in the angular direction. In the radial direction, the texture is assumed to change linearly, which is known as the rubber sheet model. The rubber sheet model linearly maps the iris texture in the radial direction from pupil border to limbus border into the interval [ $\left[\begin{array}{ll}0 & 1\end{array}\right]$ and creates a dimensionless transformation in the radial direction as well.

Although the normalization method compensates variations due to scale, translation and pupil dilation, it is not inherently invariant to the rotation of iris. Rotation of an iris in the Cartesian coordinates is equivalent to a shift in the polar coordinates. In this method, iris templates are shifted and compared in $\mathrm{n}$ different directions to compensate the rotational effects.

Here size of normalized image is $64 \times 512$

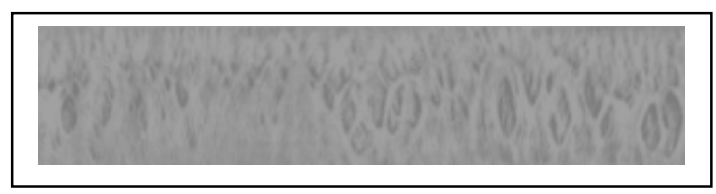

Fig. (6) Normalized iris image

\section{Enhancement}

Histogram Equalization

Histogram equalization was used for enhancement of image for getting proper intensity

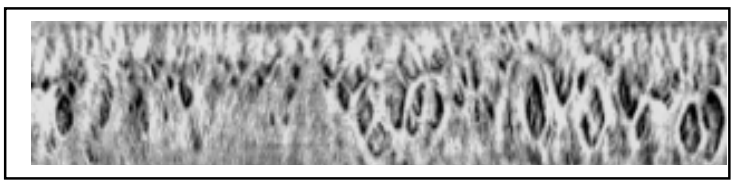

Fig. (7) Image After enhancement by histogram equalization technique

\section{Feature Extraction}

Feature extraction is a key process where the two dimensional image is converted to a set of mathematical parameters. The iris contains important unique features, such as stripes, freckles, coronas, etc. These features are collectively referred to as the texture of the iris. These features were extracted using following algorithms.

\section{Iris Code Generation}

We applied used Gaussian filter for extract the iris feature.

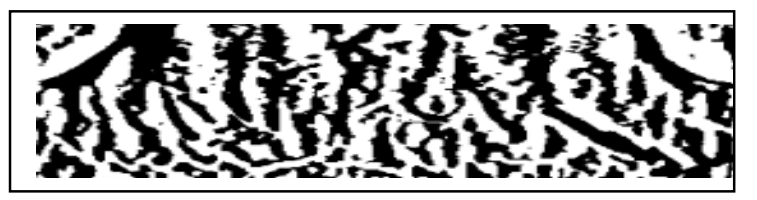

Fig .(8) Image after applying feature extraction technique.

To create iris code feature extracted image is divide into vertically 16 blocks

Step 1. Normalized image size is of $64 \times 512$. We Divide normalized iris image into basic cell regions for generation of iris code. One cell region has 64 (row) $\times 32$ (col) pixels size. A Standard deviation of pixels value is used as a representative value of a basic cell region for calculation.

Step 2. Now we got 16 bit values we have to convert this into 16 bit binary value by considering the threshold as mean from each block.

Step 3. If the pixel values of is greater than threshold make it 1.

Step 4. Else make it 0

By following above step we can obtain 16 bit binary IrisCode for Verification. 


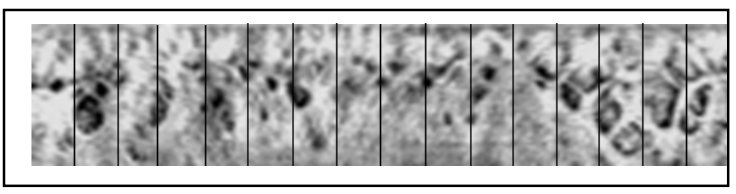

Fig. (9) Division of normalized iris image into cell regions and grouping of cell regions.

|risCode : 1 010010001111111

\section{Matching}

The pattern matching process is mainly decomposed into four parts:

- Bringing the newly acquired iris pattern into spatial alignment with a candidate database entry.

- Choosing a representation of the aligned iris pattern that makes their distinctive pattern apparent.

- Evaluating the goodness of a match between the newly acquired and database representation.

- Deciding if the newly acquired data and the database entry were derived from the same iris based on the goodness of the match.

\section{Hamming Distance}

We have used Hamming distance for finding the matches; it gives a measure of how many bits are same between two bit patterns [10]. Using the Hamming distance of two bit patterns, a decision can be made as to whether the two patterns were generated from different irises or from the same one.

$$
\mathrm{HD}=\frac{1}{N} \sum_{j=1}^{N} X_{j}(X O R) Y_{j}
$$

Since an individual iris region contains features with high degrees of freedom, each iris region will produce a bit-pattern which is independent to that produced by another iris, on the other hand, two iris codes produced from the same iris will be highly correlated.

If two bits patterns are completely independent, such as iris templates generated from different irises, the Hamming distance between the two patterns should equal 0.5. This occurs because independence implies the two bit patterns will be totally random, so there is 0.5 chance of setting any bit to 1 , and vice versa. Therefore, half of the bits will agree and half will disagree between the two patterns. If two patterns are derived from the same iris, the Hamming distance between them will be close to 0.0 , since they are highly correlated and the bits should agree between the two iris codes[12] [16].

The Hamming distance is the matching metric employed by Daugman, and calculation of the Hamming distance is taken only with bits that are generated from the actual iris region.
The Hamming distance between two strings of bits (binary integers) is the number of corresponding bit positions that differ. This can be found by using XOR on corresponding bits or equivalently, by adding corresponding bits (base 2) without a carry. For example, in the two bit strings that follow:

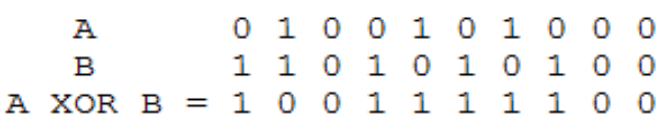

The Hamming distance $(\mathrm{H})$ between these 10-bit strings is 6 , the number of 1 's in the XOR string.

The iris is compared to previous stored iris code to compute the Hamming distance between them. Hamming distance is simply the fraction of bits that the two iris codes disagree. Hence the Hamming distance of an iris code to itself is 0 , the Hamming distance to its complement is 1 and the expected Hamming distance between 2 random iris codes is 0.5 . The Hamming distance can be computed using the elementary logical operator XOR (Exclusive-OR) and thus can be done very fast. To compensate for possible tilt of the head, the comparison is made with several different relative shifts along their angular axes [13] [14].

Here is the example of how to calculate the hamming distance between two iris codes

IrisCode1:1 100101110100011

IrisCode2:1 1000101001100101

Hamming distance of above two IrisCode is

HD : $\quad 0.3125$

Result

TABLE I. FALSE ACCEPTANCE RATE \& FALSE REJECTION RATE

\begin{tabular}{|c|l|l|l|}
\hline $\begin{array}{c}\text { HD } \\
\text { Threshold }\end{array}$ & $\begin{array}{l}\text { FAR } \\
(\%)\end{array}$ & $\begin{array}{l}\text { FRR } \\
(\%)\end{array}$ & $\begin{array}{l}\text { TSR(Total Success } \\
\text { Rate) }\end{array}$ \\
\hline 0.2 & $10 \%$ & $0 \%$ & $80 \%$ \\
\hline
\end{tabular}

\section{Conclusion}

Biometrics is the science of automated recognition of persons based on one or more physiological or behavioral characteristics. In our work we are using iris as our biometrics feature. It has importance due the very negligible change is the feature of iris. In our work we have applied Daugman's Integro- differential operator for localization, during normalization converts Cartesian to polar coordinates and histogram equalization for enhancement, in feature extraction we have used Gaussian filter and then generate the iris code. We have applied hamming distance for matching. The results were considered on MMU database. Threshold of 0.2 showed $80 \%$ success rate compared to other threshold values. 


\section{References}

[1] Sanderson S., Erbetta J.( 2000) IEE Colloquium on Visual Biometrics.

[2] Daugman J. (2002) Proceedings of International Conference on Image Processing, 1.

[3] Wolff E. (1976) Anatomy of the Eye and Orbit. $7^{\text {th }}$ edition. H. K. Lewis \& Co. LTD.

[4] Wildes R.(1997) Proceedings of the IEEE, Vol. 85, No. 9.

[5] Daugman J. (1994) Biometric personal identification system based on iris analysis. United States Patent, Patent Number: $5,291,560$.

[6] Daugman J.(1993) IEEE Transactions on Pattern Analysis and Machine Intelligence, Vol. 15, No. 11.

[7] Wildes R., Asmuth J., Green G., Hsu S., Kolczynski R., Matey J., McBride S.(1994) Proceedings IEEE Workshop on Applications of Computer Vision, Sarasota, FL, pp. 121128,.

[8] Boles W., Boashash B.( 1998) IEEE Transactions on Signal Processing, Vol. 46, No. 4.

[9] Lim S., Lee K., Byeon O., Kim T.( 2001) ETRI Journal, Vol. 23, No. 2.

[10] Umbaugh S. E. (1998) Computer Vision \& Image Processing, Prentice Hall PTR,

[11] Pizer S. M. (1987) Comput. Vision, Graphics and Image Processing, Vol. 39, pp. 355-368.

[12] Netraveli A. N. and Haskell B. G. (1988) Digital Pictures: Representation and Compression, New York: Plenum.
[13] Juwei Lu, Kostantinos N. Plataniotis, and Anasios N. Venetsanopoulos (2003) IEEE Trans. Neural Networks, vol.14, no.1.

[14] Taylor W. Change-Point Analysis: A Powerful New Tool for Detecting Changes, http://www.variation.com/cpa/tech/changepoint . $h$ tml

[15] Anil K. Jain and Jianjiang Feng(2009) IEEE TRANSACTIONS, Vol.31, No. 6.

[16] Richard O. Duda, Peter E. Hart, David G. Stork (2000) Pattern Classification, Second edition, Menlo Park, California, August.

[17] MMU Database http://pesona.mmu.edu.my/ ccteo/

[18] Rafael C. Gonzalez, Richard E. Eoods, Steven L. Eddins," Digital Image Processing Using Matlab" 